\title{
A multi-center study on the risk factors of infection caused by multi-drug resistant Acinetobacter baumannii
}

Huiping Huang ${ }^{1 \dagger}$, Borong Chen ${ }^{2 \dagger}$, Gang Liu ${ }^{2 \dagger}$, Jing Ran ${ }^{3}$, Xianyu Lian ${ }^{1}$, Xinhua Huang ${ }^{4}$, Nan Wang ${ }^{5}$ and Zhengjie Huang ${ }^{2^{*}}$

\begin{abstract}
Background: Acinetobacter baumannii (AB) is critical for healthcare-associated infections (HAl) with significant regional differences in the resistance rate, but its risk factors and infection trends has not been well studied. We aimed to explore the risk factors, epidemiological characteristics and resistance of multidrug-resistant Acinetobacter baumannii (MDR-AB) in intensive care unit inpatients.

Methods: Data of patients with MDR-AB (195 cases), and with antibiotic-sensitive AB infection (294 cases, control) during January to December, 2015 in three medical centers in Xiamen, China were conducted and analyzed in the present retrospective study.

Results: Lower respiratory tract infection with AB accounted for $68.71 \%$. MDR-AB was detected in $39.88 \%$ of all cases. Univariate analysis suggested that mechanical ventilation, indwelling catheter, cancer patients, length of hospitalization in intensive care unit (ICU) $\geq 15 \mathrm{~d}$, Acute Physiology and Chronic Health Evaluation (APACHE) ॥ score, combined using antibiotic before isolation of $A B$ and use of third-lines cephalosporins were associated with the development of MDR-AB healthcare-associated infections. Dose-response relationship analysis suggested that the age and the days of mechanical ventilation were associated with increased infection with MDR-AB. Logistic regression analysis suggested that, mechanical ventilation, combined using antibiotic before isolation of $A B$, and indwelling catheter, were associated with MDR-AB infection, with odds ratios $(O R)$ and $95 \%$ confidence intervals (CI) of 3.93 (1.52-10.14), 4.11 (1.58-10.73), and 4.15 (1.32-12.99), respectively.

Conclusions: MDR-AB infection was associated with mechanical ventilation, combined using antibiotic before isolation of $A B$, and indwelling catheter. Furthermore, the age and the days of mechanical ventilation were associated with increased infection with MDR-AB.
\end{abstract}

Keywords: Intensive care unit, Multi-drug resistant, Acinetobacter baummanii, Risk factors

\section{Background}

Acinetobacter baumannii (AB) is a Gram-negative, lactose non-fermenting organism and its ability to survive in hospital environments, which is increasingly becoming a major healthcare-associated infections (HAI) pathogen worldwide. With the emergence of $\mathrm{HAI}, \mathrm{AB}$ is an important cause in critically ill patients. $A B$ is now largely

\footnotetext{
* Correspondence: huangzhengjie@xmu.edu.cn

${ }^{\dagger}$ Equal contributors

${ }^{2}$ Department of Gastrointestinal Surgery, Xiamen Cancer Hospital, The First Affiliated Hospital of Xiamen University, 55 Zhen Hai Road, Si Ming District, Xiamen, Fujian 361003, China

Full list of author information is available at the end of the article
}

regarded as one of the most troublesome pathogens and is responsible for several types of HAI including skin and soft tissue infections and invasive infections, such as pneumonia, osteomyelitis, and bacteremia [1].

Reports about multi-drug resistance Acinetobacter baumannii (MDR-AB) has been constantly increased, especially in the intensive care units (ICU) [2-4]. MDR$\mathrm{AB}$-caused infections are difficult to diagnose and treat, leading to increased mortality and prolonged hospital stays [5]. A recent study demonstrated that the 30-day hospital mortality rate of bloodstream infections caused by MDR-AB was $55.2 \%$ in geriatric inpatients [1]. 
Carbapenem resistant rate increases every year. It was showed that some hospital ICU emerged carbapenem resistant (CR) AB. Resistance of AB isolates to imipenem dramatically increased from $16 \%$ in $2003-2007$ to $78 \%$ in 2008-2010 in a single tertiary hospital in South Korea $[6,7]$. Cross-transmission of MDR-AB was common in the hospital, resulting in ICU and neonatal ward infection outbreaks and epidemics, and was correlated with an adverse outcome, including an independent predictor of death and complications [8-11].

A large amount of data is available regarding the epidemiology, risk factors, and outcomes of patients with AB. However, besides the rapid growth worldwide, there are significant regional differences in the resistance rate of $\mathrm{AB}$. Information is limited regarding the risk factors for MDR-AB in ICU inpatients in developing countries.

Therefore, we designed the present retrospective study to investigate the risk factors and infection trends caused by MDR-AB in ICU inpatients. Patients in three university affiliated hospitals with more than 800 beds in Xiamen, China, were evaluated. The clinical characteristics as well as the trends of MDR-AB with the dose-response relationship were analyzed. Our study is beneficial for understanding clinical significance and risk factors of MDR-AB, and for providing support for future management in ICU clinical practice.

\section{Methods}

\section{Setting}

Three ICU of tertiary general hospitals in Xiamen, including the First Affiliated Hospital of Xiamen University, the No. 2 Hospital of Xiamen, and 174th Hospital of the Chinese People's Liberation Army (the Affiliated Chenggong Hospital of Xiamen University), of which the microbial identification results are homogeneous and are regulatory accepted by each other, were selected by using stratified sampling method. Four hundred eighty-nine patients who were hospitalized during the period of January 1st to December 31st, 2015 patients with $\mathrm{AB}$ infection were included as participants in the present study. Repeated strains isolated from the same patient in the same part of the specimen were excluded.

The quality control methods of sputum samples: Under laboratory microscope, if the squamous epithelial cells in the low fold field of vision $<10$ and the white blood cells $>25$ are qualified samples.

Diagnostic criteria: The designation of MDR was defined as the absence of susceptibility to $>3$ of the following antimicrobials or groups of antimicrobials: ampicillin/sulbactam, aztreonam, ceftazidime, ciprofloxacin, gentamicin, imipenem, piperacillin, trimethoprim/sulfamethoxazole, carbapenems, and amikacin $[12,13]$.Bacterial isolation and antimicrobial susceptibility testing were performed in accordance with the methodology of the Clinical and Laboratory Standards Institute [14]. HAI was confirmed according to the surveillance definition of the Centers for Disease Control and Prevention/National Healthcare Safety [15].

\section{Investigation methods}

The cases were reviewed in the microbiology and inspection laboratory database by searching for at least 2 positive cultures. Through a retrospective survey, the medical records of the cases were obtained from the medical record archives. The demographics, information, regarding clinical care, microbiologic data, treatments provided, and outcomes of the patients with clinically significant $\mathrm{AB}$ were reviewed.

Main contents of questionnaire including the name, gender, age, APACHE II score, comorbidities, ICU days, infection site, HAI, hospitalization days before infection, operation, invasive operation, antibiotic use, and drug sensitivity were collected. The data were analyzed after double-entry.

\section{Definitions of main risk factors}

1) Comorbidities refer to hypertension, coronary heart disease, diabetes, cancer, chronic renal insufficiency, cerebral infarction, etc. 2) ICU days refers to patients with ICU admission until test positive for the first time. 3) The combination of antimicrobial agents is the use of 2 or more than 2 kinds of antibacterial drugs. 4) Invasive operation refers to tracheotomy, nasal feeding, indwelling catheter, arteriovenous catheter, abdominal puncture, ventilator etc.

\section{Statistical analysis}

All of the statistical analyses were performed using SPSS 13.0 (SPSS Inc., Chicago, IL, USA). Categorical variables were analyzed using the $\mathrm{X}^{2}$ test or Fisher's exact test, and continuous variables were analyzed using Student's t test or Mann-Whitney $U$ test, and were generally presented as means and standard deviation. Multivariate logistic regression analyses using the forward likelihood ratio selection method were used. To identify independent factors of MDR-AB and is presented with an odds ratio ( $95 \%$ confidence intervals, CI). Potential candidate variables were those with $P<0.05$ in univariate analyses. All of the $P$ values were 2 tailed, and $P<0.05$ was considered statistically significant.

\section{Results}

\section{Clinical characteristics}

A total of 489 patients aged from 38 days to 101 years with $\mathrm{AB}$ isolations during January to December 2015 were identified in this study. The mean age of the patients was $56.54 \pm 27.18$ years. The male to female ratio was 3.9:1(Male 390, female 99). Two hundred twenty-one of the 
Table 1 Source of Acinetobacter baummanii from clinical specimens

\begin{tabular}{|c|c|c|c|c|c|}
\hline \multirow[t]{2}{*}{ Group } & Sputum & Whole blood & Drainage fluid & Central venous catheter tip & Others \\
\hline & $\mathrm{N} \%$ & N\% & N\% & $\mathrm{N} \%$ & N\% \\
\hline MDR-AB & $141(72.3)$ & $9(4.6)$ & $21(10.8)$ & $10(5.1)$ & $14(7.2)$ \\
\hline Non-MDR-AB & $195(66.3)$ & $23(7.8)$ & $29(9.9)$ & $12(4.1)$ & $35(11.9)$ \\
\hline Total & 336 (68.7) & $32(6.5)$ & $50(10.2)$ & $22(4.5)$ & $49(10.0)$ \\
\hline
\end{tabular}

$\mathrm{X}^{2}=5.45, P=0.24$

cases were healthcare-associated infections, accounting for 45.20\%.

MDR-AB was identified in up to 195 cases (39.88\%), male 165 and female 30. Two hundred ninety-four cases were Non-MDR-AB, male 225 and female 69. The gender was significantly associated with the infection caused by MDR-AB $\left(\mathrm{X}^{2}=4.74, P=0.03\right)$.

The average age of the patients $(64.93 \pm 21.13)$ was significantly higher than that $(50.98 \pm 29.28)$ in the control group $(t=5.74, P<0.001)$.

Table 2 The results of one-way Chi-square test about exposure factors in MDR-AB infection patients

\begin{tabular}{|c|c|c|c|c|}
\hline \multirow[t]{2}{*}{ Characteristics } & & \multicolumn{2}{|c|}{ Acinetobacter baumannii bacteremia N (\%) } & \multirow[t]{2}{*}{$P$} \\
\hline & & $\begin{array}{l}\text { MDR-AB } \\
(N=195)\end{array}$ & $\begin{array}{l}\text { Non-MDRAB } \\
(N=294)\end{array}$ & \\
\hline \multirow[t]{2}{*}{ Sex } & Male & $165(84.6)$ & $225(76.5)$ & 0.03 \\
\hline & Female & $30(15.4)$ & $69(23.5)$ & \\
\hline \multirow[t]{5}{*}{ Age (y) } & $<50$ & $10(5.13)$ & $58(19.7)$ & \\
\hline & $50-60$ & $22(11.3)$ & $131(44.6)$ & $<0.001$ \\
\hline & $60-70$ & $98(50.3)$ & $62(21.1)$ & \\
\hline & $70-80$ & $46(23.6)$ & $34(11.6)$ & \\
\hline & $>80$ & $19(9.74)$ & $9(3.06)$ & \\
\hline \multirow[t]{2}{*}{ Recent history of surgery } & Yes & $54(27.7)$ & $75(25.5)$ & 0.59 \\
\hline & No & $141(72.3)$ & $219(74.5)$ & \\
\hline \multirow[t]{2}{*}{ Comorbidities } & $<2$ & $165(84.6)$ & $246(83.7)$ & 0.78 \\
\hline & $\geq 2$ & $30(15.4)$ & $48(16.3)$ & \\
\hline \multirow[t]{2}{*}{$\mathrm{HAl}$} & Yes & $117(60.0)$ & $104(35.4)$ & $<0.001$ \\
\hline & No & $78(40.0)$ & $190(64.6)$ & \\
\hline \multirow[t]{2}{*}{ ICU days } & $<15 d$ & $24(12.3)$ & $78(26.5)$ & $<0.001$ \\
\hline & $\geq 15 d$ & $171(87.7)$ & $216(73.5)$ & \\
\hline \multirow[t]{2}{*}{ Mechanical ventilation } & Yes & $177(90.8)$ & $210(71.4)$ & $<0.001$ \\
\hline & No & $18(9.2)$ & $84(28.6)$ & \\
\hline \multirow[t]{2}{*}{ Indwelling catheters } & Yes & $183(93.8)$ & $234(79.6)$ & $<0.001$ \\
\hline & No & $12(6.2)$ & $60(20.4)$ & \\
\hline \multirow[t]{2}{*}{ Combined use of antimicrobial agents prior to infection } & Yes & $174(89.2)$ & $246(83.7)$ & $<0.001$ \\
\hline & No & $21(10.8)$ & $48(16.3)$ & \\
\hline \multirow[t]{2}{*}{ Use of third-generation cephaloglycin } & Yes & $186(95.4)$ & $246(83.7)$ & $<0.001$ \\
\hline & No & $9(4.6)$ & $48(16.3)$ & \\
\hline \multirow[t]{2}{*}{ Hospitalization times } & $<3$ & $33(16.9)$ & 97 (33.0) & $<0.001$ \\
\hline & $\geq 3$ & $162(83.1)$ & $197(67.0)$ & \\
\hline \multirow[t]{2}{*}{ History of cancer } & Yes & $121(62.1)$ & $148(50.3)$ & 0.01 \\
\hline & No & 74 (37.9) & $146(49.7)$ & \\
\hline \multirow[t]{2}{*}{ APACHE II score } & $<18$ & $117(60.0)$ & $228(77.6)$ & $<0.001$ \\
\hline & $\geq 18$ & $78(40.0)$ & $66(22.4)$ & \\
\hline
\end{tabular}


Table 3 The results of unconditional logistic multiple regression analysis about the risk factors of MDR-AB infection

\begin{tabular}{|c|c|c|c|c|c|c|}
\hline Risk factors & $B(k)$ & SE (B) & $x^{2}$ & $P$ & $O R$ & $95 \% \mathrm{Cl}$ \\
\hline Mechanical ventilation & 1.37 & 0.48 & 8.03 & $<0.001$ & 3.93 & $1.53-10.14$ \\
\hline Combined use of Antimicrobial agents & 1.41 & 0.49 & 8.35 & $<0.001$ & 4.11 & $1.58-10.73$ \\
\hline Indwelling catheter & 1.42 & 0.58 & 5.97 & 0.02 & 4.15 & $1.33-13.00$ \\
\hline
\end{tabular}

The positive $\mathrm{AB}$ isolations were common in the respiratory tract $(72.31 \%$ versus $66.33 \%$, respectively). There was no significant difference between the two groups (Table 1).

\section{Univariate analysis for risk factors of MDR-AB}

The $\mathrm{X}^{2}$ analysis results show, HAI, mechanical ventilation, indwelling catheters, ICU days, APACHE II score, combined use of antimicrobial agents prior to infection and the use of third-generation cephalosporins, hospitalization $\geq 3$ times, history of cancer are associated with MDR-AB infection. And the operation history, comorbidities and other factors are no significant correlated with MDR-AB infection (Table 2).

\section{Multivariate regression analysis results}

An unconditional logistic multiple regression analysis was performed on 9 variables with significant differences in single factor analysis. Finally logistic multiple regression analysis revealed that the mechanical ventilation, combined use of antibacterial drugs before infection, and indwelling catheter were independent risk factors associated with the MDR-AB infection, respectively (Table 3 ).

\section{Dose-response relationship}

The trend $\mathrm{X}^{2}$ analysis showed that there was a doseresponse relationship between the age, the days of mechanical ventilation, and MDR-AB infections, respectively. The age and the days of mechanical ventilation are both positively associated with the MDR-AB infections (Table 4).

\section{Discussion}

MDR-AB infection could significantly prolong the hospital stay, increase mortality, and increase economic costs $[8,11]$. The present study was a retrospective, observational, multi-center study. Multiple regression analysis was employed to minimize the confounding bias. We showed that the incidence of MDR-AB was $39.88 \%$ in ICU inpatients, and was less than $56.3 \%$, while more than $50 \%$ of the positive $A B$ specimens were from sputum and lower respiratory tract infection was predominant. Our results were in concert with the studies reported by Zorgan, Dejsirilert, Custovic, etc. [2, 16-19]. In addition, we analyzed the relationship between the use of antibacterial agents and MDR-AB infection. It is found that combined use of antibiotics before the infection is an important factor affecting the incidence of MDR-AB.

Studies had shown that tumor history, high APACHE II score and indwelling catheter were risk factors with the infection of resistant bacteria [9, 20, 21]. By using univariate analysis, we also found that the risk of MDR-AB infection in the patients with hospitalization $\geq 3$ times was 2.42 fold of that in patients with hospitalization $<3$ times.

In our study, a significant association between MDR$\mathrm{AB}$ infection and mechanical ventilation, combined use of antimicrobial agents, indwelling catheters were noted. There were independent risk factors for MDR$\mathrm{AB}$ in ICU inpatients. Mechanical ventilation is often considered as an important factor in the MDR Acinetobacter pneumonia $[4,22,23]$. In the present study,

Table 4 The results of tendency chi-square test about the relation of MDR-AB infection with the age and the days of mechanical ventilation

\begin{tabular}{|c|c|c|c|c|c|c|}
\hline Exposure factors & Observed cases & Cases of MDR-AB & Infection rate (\%) & OR & $x^{2}$ & $P$ \\
\hline \multicolumn{7}{|l|}{ Age (years) } \\
\hline 0 & 13 & 2 & 15.38 & 1.00 & \multirow[t]{5}{*}{58.62} & \multirow[t]{5}{*}{$<0.001$} \\
\hline 20 & 69 & 16 & 23.19 & 1.36 & & \\
\hline 40 & 127 & 37 & 29.13 & 1.41 & & \\
\hline 60 & 184 & 72 & 39.13 & 1.64 & & \\
\hline 80 & 96 & 68 & 70.83 & 3.43 & & \\
\hline \multicolumn{7}{|c|}{ Mechanical ventilation (days) } \\
\hline 0 & 102 & 18 & 17.65 & 1.00 & \multirow[t]{3}{*}{43.36} & \multirow[t]{3}{*}{$<0.001$} \\
\hline 10 & 247 & 94 & 38.06 & 2.87 & & \\
\hline 20 & 140 & 83 & 59.29 & 6.80 & & \\
\hline
\end{tabular}


mechanical ventilation was in 187 of 195 cases (accounting for $95.89 \%$ ), whereas lower respiratory tract infections accounted for $72.31 \%$, further confirmed mechanical ventilation as an important factor for MDR-AB infection.

The use of multiple types of antibiotics also increased MDR-AB infections $(O R=4.111)$, since we also found that combination with antibiotics before infection is an important risk factor. Studies have reported that, use of carbapenem antibiotics within 28 days of infection was significantly related with MDR bacterial infection $[21,23,24]$. Inappropriate drug combination leads to selective pressure, which increases the opportunity of $\mathrm{AB}$ infection and promotes the selection of drugresistant bacteria. The $\beta$ - lactam antimicrobial drugs could induce $A B$ to produce $\beta$-lactamase, and hence inactivate antimicrobial drugs, resulting in resistance of $A B$ to other $\beta$-lactam antibiotics [25]. Therefore, the principle of the combination must be strictly followed, and the frequent replacement of antibiotics should be avoided.

Indwelling catheter is also an independent risk factor for MDR-AB infection (OR = 4.149). Similar results were also reported previously [21, 22]. MDR-AB could spread through catheter and puncture point, and treatment device.

The trend $\mathrm{X}^{2}$ analysis showed that there was a significant dose-response relationship between age, days of mechanical ventilation, and MDR-AB infections. The risk of MDR-AB infections increased with higher age, and with longer time of mechanical ventilation.

\section{Conclusions}

In conclusion, MDR-AB infection was associated with mechanical ventilation, combined using antibiotic before isolation of $\mathrm{AB}$, and indwelling catheter. Furthermore, the age and the days of mechanical ventilation were associated with increased infection with MDR-AB.

\section{Abbreviations}

AB: Acinetobacter baumannii;; APACHE: Acute Physiology and Chronic Health Evaluation; Cl: Confidence intervals; CR: Carbapenem resistant; HAl: Healthcareassociated infections; ICU: Intensive care unit; MDR-AB: Multidrug-resistant Acinetobacter baumannii; OR: Odds ratios

\section{Acknowledgements}

We would like to thank everyone who helped with this study.

\section{Funding}

This study was supported by grants from the Science and Technology Project of Natural Science Foundation of Fujian Province (No. 2016 J01639, 2016 J01640), the Medical Innovations Topic in Fujian Province (No. 2016-CXB-8, 2012-CXB-29) and Project of Xiamen Scientific and Technological Plan (No. 3502Z20134011). They have not influenced the study design, data collection and analysis, and preparation of the manuscript.
Availability of data and materials

Restrictions apply to the availability of these data, which were used under license for the current study, and so are not publicly available. Data are, however, available from the authors upon reasonable request.

\section{Authors' contributions}

$\mathrm{ZJH}$ and $\mathrm{HPH}$ conceptualized and designed the study. BRC, GL, JR, XYL, XHH and NW did data collection and input. ZJH and HPH checked and review data. BRC and GL performed the statistical analysis. HPH, BRC and GL drafted the manuscript. ZJH contributed to revise the manuscript. All authors read and approved the final manuscript.

\section{Ethics approval and consent to participate}

The Ethics Committee of the First Affiliated Hospital of Xiamen University, the Ethics Committee of the Second Hospital of Xiamen and the Ethics Committee of the Chenggong Hospital Affiliated to Xiamen University approved this study. The matched clinical information was collected and analyzed with each participant written informed consent. Meanwhile, for participants under the age of 16 , the consent was obtained from a parent or guardian on behalf. All participants consented that the matched clinical information would be submitted for publication.

Consent for publication

Not applicable.

\section{Competing interests}

The authors declare that they have no competing interests.

\section{Publisher's Note}

Springer Nature remains neutral with regard to jurisdictional claims in published maps and institutional affiliations.

\section{Author details}

'Department of Infection Control, The First Affiliated Hospital of Xiamen University, Xiamen, Fujian 361003, China. ${ }^{2}$ Department of Gastrointestinal Surgery, Xiamen Cancer Hospital, The First Affiliated Hospital of Xiamen University, 55 Zhen Hai Road, Si Ming District, Xiamen, Fujian 361003, China. ${ }^{3}$ Department of Gynecology and Obstetrics, The First Affiliated Hospital of Xiamen University, Xiamen, Fujian 361003, China. ${ }^{4}$ Department of Infection Control, The Second Hospital of Xiamen, Xiamen, Fujian 361021, China. ${ }^{5}$ Department of Infection Control, The Affiliated Chenggong Hospital of Xiamen University, Xiamen, Fujian 361003, China.

Received: 6 August 2017 Accepted: 19 December 2017

Published online: 05 January 2018

\section{References}

1. Fu Q, Ye H, Liu S. Risk factors for extensive drug-resistance and mortality in geriatric inpatients with bacteremia caused by Acinetobacter baumannii. Am J Infect Control. 2015;43(8):857-60.

2. Chaisathaphol T, Chayakulkeeree M. Epidemiology of infections caused by multidrug-resistant gram-negative bacteria in adult hospitalized patients at Siriraj Hospital. J Med Assoc Thail. 2014;97(Suppl 3):S35-45.

3. Behnia M, et al. Nosocomial and ventilator-associated pneumonia in a community hospital intensive care unit: a retrospective review and analysis. BMC Res Notes. 2014;7:232.

4. Medell $\mathrm{M}$, et al. Nosocomial ventilator-associated pneumonia in Cuban intensive care units: bacterial species and antibiotic resistance. MEDICC Rev. 2013;15(2):26-9.

5. Perez F, et al. Global challenge of multidrug-resistant Acinetobacter baumannii. Antimicrob Agents Chemother. 2007;51(10):3471-84.

6. Werarak $\mathrm{P}$, et al. Acinetobacter baumannii nosocomial pneumonia in tertiary care hospitals in Thailand. J Med Assoc Thail. 2012;95(Suppl 2):S23-33.

7. Gurung $\mathrm{M}$, et al. Emergence and spread of carbapenem-resistant Acinetobacter baumannii sequence type 191 in a Korean hospital. Infect Genet Evol. 2013;19:219-22.

8. Garlantezec R, et al. Cost-analysis of an intensive care unit closure due to an imipenem-resistant oxa-23 Acinetobacter baumannii outbreak. J Hosp Infect. 2011;77(2):174-5. 
9. Zarrilli $\mathrm{R}$, et al. Clonal spread and patient risk factors for acquisition of extensively drug-resistant Acinetobacter baumannii in a neonatal intensive care unit in Italy. J Hosp Infect. 2012;82(4):260-5.

10. Agodi A, et al. Spread of a carbapenem- and colistin-resistant Acinetobacter baumannii ST2 clonal strain causing outbreaks in two Sicilian hospitals. J Hosp Infect. 2014;86(4):260-6.

11. Ayraud-Thevenot $\mathrm{S}$, et al. Control of multi-drug-resistant Acinetobacter baumannii outbreaks in an intensive care unit: feasibility and economic impact of rapid unit closure. J Hosp Infect. 2012;82(4):290-2.

12. Zarrilli $R$, et al. Global evolution of multidrug-resistant Acinetobacter baumannii clonal lineages. Int J Antimicrob Agents. 2013;41(1):11-9.

13. Taitt $C R$, et al. Antimicrobial resistance determinants in Acinetobacter baumannii isolates taken from military treatment facilities. Antimicrob Agents Chemother. 2014;58(2):767-81.

14. Institute, C.a.L.S. Methods for dilution antimicrobial susceptiblity tests for bacteria that grow aerobically;approved standard-eighth edition. Clinical and Laboratory Standards Institute. 2008;29(2):11.

15. Horan TC, Andrus M, Dudeck MA. CDC/NHSN surveillance definition of health care-associated infection and criteria for specific types of infections in the acute care setting. Am J Infect Control. 2008;36(5):309-32.

16. Zorgani A, et al. Prevalence of device-associated Nosocomial infections caused by gram-negative bacteria in a trauma intensive care unit in Libya. Oman Med J. 2015;30(4):270-5

17. Dejsirilert $\mathrm{S}$, et al. Antimicrobial resistance of Acinetobacter baumannii: six years of National Antimicrobial Resistance Surveillance Thailand (NARST) surveillance. J Med Assoc Thail. 2009:92(Suppl 4):S34-45.

18. Custovic A, et al. Epidemiological monitoring of nosocomial infections caused by Acinetobacter baumannii. Med Arch. 2014;68(6):402-6.

19. Koo SH, et al. Genetic basis of multidrug-resistant Acinetobacter baumannii clinical isolates from three university hospitals in Chungcheong Province, Korea. Korean J Lab Med. 2010;30(5):498-506.

20. Perez F, Adachi J, Bonomo RA. Antibiotic-resistant gram-negative bacterial infections in patients with cancer. Clin Infect Dis. 2014;59(Suppl 5):S335-9.

21. Vasudevan A, et al. Risk factors for infection/colonization caused by resistant Gram negative bacilli in critically ill patients (an observational study of 1633 critically ill patients). Prev Med. 2013;57(Suppl):S70-3.

22. Jung JY, et al. Risk factors for multi-drug resistant Acinetobacter baumanni bacteremia in patients with colonization in the intensive care unit. BMC Infect Dis. 2010;10:228.

23. Zheng $Y L$, et al. Risk factors and mortality of patients with nosocomial carbapenem-resistant Acinetobacter baumannii pneumonia. Am J Infect Control. 2013:41(7):e59-63.

24. McCracken M, et al. Characterization of Acinetobacter baumannii and meropenem-resistant Pseudomonas aeruginosa in Canada: results of the CANWARD 2007-2009 study. Diagn Microbiol Infect Dis. 2011;69(3):335-41.

25. Roca I, et al. The Acinetobacter baumannii oxymoron: commensal hospital dweller turned pan-drug-resistant menace. Front Microbiol. 2012;3:148

\section{Submit your next manuscript to BioMed Central and we will help you at every step:}

- We accept pre-submission inquiries

- Our selector tool helps you to find the most relevant journal

- We provide round the clock customer support

- Convenient online submission

- Thorough peer review

- Inclusion in PubMed and all major indexing services

- Maximum visibility for your research

Submit your manuscript at www.biomedcentral.com/submit 